${ }^{1}$ Departament of Orthodontics, Araras Dental School, Uniararas, Araras, SP, Brazil.

${ }^{2}$ Department of Restorative Dentistry - Dental Materials Division, Piracicaba Dental School, Unicamp, Piracicaba, SP, Brazil.
Corresponding author: Heloisa C Valdrighi Av. Dr. Maximiliano Baruto, 500 Jardim Universitário. Araras, SP - Brazil. 13607-339

19 3543-1423

e-mail: heloisavaldrighi@gmail.com (iD) https://orcid.org/0000-0001-7567-1990

Received: May 03, 2019

Accepted: October 19, 2019

\section{Can the chlorhexidine gluconate solution potentiate the staining of polycrystalline ceramic brackets?}

Catharina e Silva Monteiro Barros ${ }^{1}$, Jose

Guilherme Neves ${ }^{2}$, Ana Paula Terossi De Godoi ${ }^{1}$, Ana Rosa Costa ${ }^{2}$, Mario Vedovello Filho ${ }^{1}$, Heloisa Cristina Valdrighi ${ }^{1, *}$

Aim: The present study aimed to assess, in vitro, the effect of chlorhexidine on the potentiation of polycrystalline ceramic bracket staining. Methods: Seventy-two polycrystalline ceramic brackets of upper right central incisors were divided into six groups $(n=12)$ according to immersion solution. The groups were G1 - distilled water (control); G2 - chlorhexidine; G3 - coffee; G4 - red wine; G5 - chlorhexidine associated with coffee; and G6 - chlorhexidine associated with red wine. The samples were analyzed by means of a spectrophotometer according to the $\mathrm{CIEL}{ }^{*}{ }^{*} \mathrm{~b}^{*}$ system, and color change $\left(\triangle \mathrm{E}^{*}\right)$ was calculated. The readings were performed at the following times: T0 - After package removal and T1 - After staining. The data were analyzed by Kruskal Wallis and t tests $(p<0.05)$ at $5 \%$ significance level. Results: The total color variation $\left(\Delta \mathrm{E}^{\star}\right)$ was greater in the group that received chlorhexidine associated with red wine $(p<0.05)$ and lower in the groups that received distilled water. All other groups showed greater value variations when compared to G1 and G2. Group G6 showed a greater color change due to the potentiation of chlorhexidine with the dye substance. Conclusion: It is concluded that chlorhexidine potentiates the staining caused by red wine in polycrystalline ceramic brackets.

Keywords: Orthodontic brackets. Mouthwashs. Ceramics. 


\section{Introduction}

Over the last years, patients seeking orthodontic treatment have not only been demanding an effective occlusal result, but also looking for the satisfaction of a harmonious appearance, aiming to resemble the color of tooth enamel1,2. The use of esthetic devices is mainly indicated for adult patients who wish to maintain a natural aspect to their smile and correct its positioning at the same time ${ }^{3}$.

The first esthetic orthodontic devices were polycarbonate brackets, which present negative characteristics such as high deformation rate, structural fragility, low adhesion, and low stain resistance, thus compromising clinical performance and not reaching the esthetic objective ${ }^{4}$.

Attempting to solve such problems, a few structural changes were performed, such as the reinforcement with ceramic loads and glass fiber, which originated the esthetic ceramic brackets ${ }^{5}$. According to the fabrication process, there are two forms of ceramic brackets: polycrystalline and monocrystalline ${ }^{6}$. The polycrystalline ceramic or polycrystalline alumina brackets are made of aluminum oxide crystals fused at high temperatures that allow molding several brackets simultaneously. Among esthetic brackets, these are the most common and popular, due to material quality and the ease of production in comparison to monocrystalline alumina brackets ${ }^{7}$.

The ceramic brackets, although desired by patients, still deteriorate in the oral cavity and present some limitations when exposed to certain substances such as coffee, black tea, and red wine, causing color change and consequently the loss of the esthetic standard desired ${ }^{8-10}$. This impregnation of food and beverage pigments is considered one of the main extrinsic staining factors of such devices ${ }^{11}$.

In the clinical procedure during orthodontic treatment, the use of mouthwashes is rather common ${ }^{12}$. However, few studies show the effects of mouthwashes on the color stability of polycrystalline ceramic orthodontic brackets ${ }^{13}$ and composite resins $^{14}$. Chlorhexidine gluconate, from the biguanide family, is considered the gold standard in therapies for controlling plaque ${ }^{15}$, thus preventing potential inflammation of the periodontal tissue adjacent to the orthodontic appliances. On the other hand, the continuous use of chlorhexidine can generate the appearance of dark spots on dental surfaces, presenting variation according to the frequency of use and concentration of the product used ${ }^{15}$.

Due to the presence of pigmentation in esthetic orthodontic brackets caused by the consumption of coloring foods and mouthwashes, the present study aimed to assess, in vitro, the effect of chlorhexidine on the potentiation of ceramic bracket staining. The null hypothesis in this study was that chlorhexidine does not have the capacity to potentiate staining in ceramic brackets

\section{MATERIAL AND METHODS}

The sample size considered the previous literature ${ }^{9,13}$, significance level of 0.05 , and test power of 0.80 . Therefore, the final sample included 72 polycrystalline ceramic brackets (Iceram, Orthometric, Marília, São Paulo, Brazil; Batch 037268001) of upper 
right central incisors, which were divided into six groups $(n=12)$ according to dye solution: G1 - distilled water (control group, Asfer, Indústria Química, São Caetano do Sul, SP, Brazil; Batch 274); G2 - 0.12\% chlorhexidine (Periogard, Colgate; Batch 7125BR121A); G3 - instant coffee (Nescafé, Nestlé, São Paulo, Brazil; Batch 80301210); G4 - red wine (Reservado, Carbenet Sauvignon 12.5\% alcohol volume, Concha y Toro/ Santiago do Chile; Batch: 1110716); G5 - chlorhexidine and coffee; G6 - chlorhexidine and wine, as Figure 1 shows.

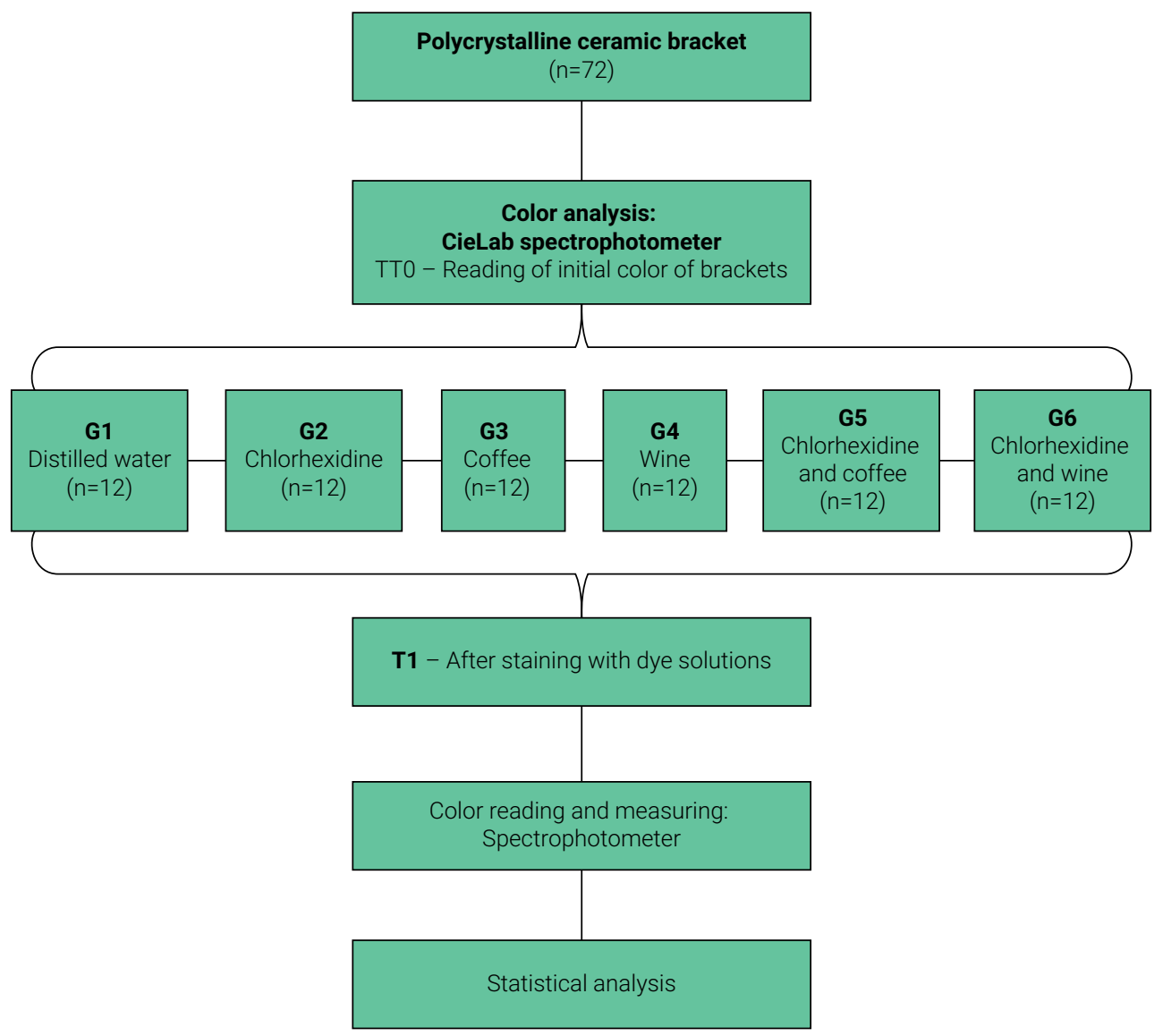

Figure 1. Experimental design of the study.

\section{PIGMENTATION PROCESS}

The instant coffee solution was prepared according to the concentration suggested by the manufacturer, with $100 \mathrm{ml}$ of boiling water to two teaspoons of powder. The other solutions did not require previous preparations.

The specimens were immersed in staining solutions and maintained under agitation (ultrasonic tank) according to each group described. The samples were immersed for three minutes, once a day for five days of the week during 28 days ${ }^{16-18}$. Then, the solu- 
tions were removed and the specimens were washed with distilled water and dried with absorbent paper. Next, the specimens were stored in distilled water at $37^{\circ} \mathrm{C}$ until the next staining protocol ${ }^{18,19}$. On the other hand, the control group remained in distilled water for the entire experimental period. All groups were immersed in $3 \mathrm{ml}$ of each dye solution, stored in units in a lidded polypropylene recipient to prevent the evaporation of solutions and identify the group name in order to to codify it for individualization.

\section{COLOR READING}

The samples were subjected to color reading using a SP62S X-Rite spectrophotometer (Grand Rapids, Michigan, USA) with the QA Master software. This reading was performed at the times described, that is, initial time (TO), immediately after package removal, immediately after water storage, and at the end of the entire pigmentation process (T1).

This spectrophotometer presents focal aperture of $4 \mathrm{~mm}$ and diffuse geometry of $\mathrm{D} / 8^{\circ}$. It emits light with wavelength from 400 to $700 \mathrm{~nm}$ on the object and measures the reflection of such spectrum. Color was measured with the CIEL*a*b* color system. The $\Delta \mathrm{E}^{\star}$, that is, the total difference between two color stimuli, was calculated with the following formula: $\Delta \mathrm{E}^{\star}=\sqrt{ }\left(\Delta \mathrm{L}^{*}\right)^{2}+\left(\Delta \mathrm{a}^{*}\right)^{2}+\left(\Delta \mathrm{b}^{\star}\right)^{2}$.

For the color readings, the brackets were supported on a white surface properly standardized for the reading so it was not affected by the background color. For the readings, the brackets were manipulated with clinical tweezers and procedure gloves. The positioning for the reading was always the center of the spectrophotometer, with the incisal side toward the center of the device and the buccal surface upward, and it was determined by tagging the bracket to standardize the position for color reading in the different axes $\left(L^{*} a^{\star} b^{\star}\right)$. The $L^{*}$ parameter corresponds to the value or degree of lightness or brightness and $a^{*} b^{\star}$ values of chroma, where $+a^{*}$ is red and $-a^{*}$ is green, $+b^{*}$ is yellow and $-b^{*}$ is blue.

This process was performed once per bracket and time, always repositioning the bracket in the center of the device.

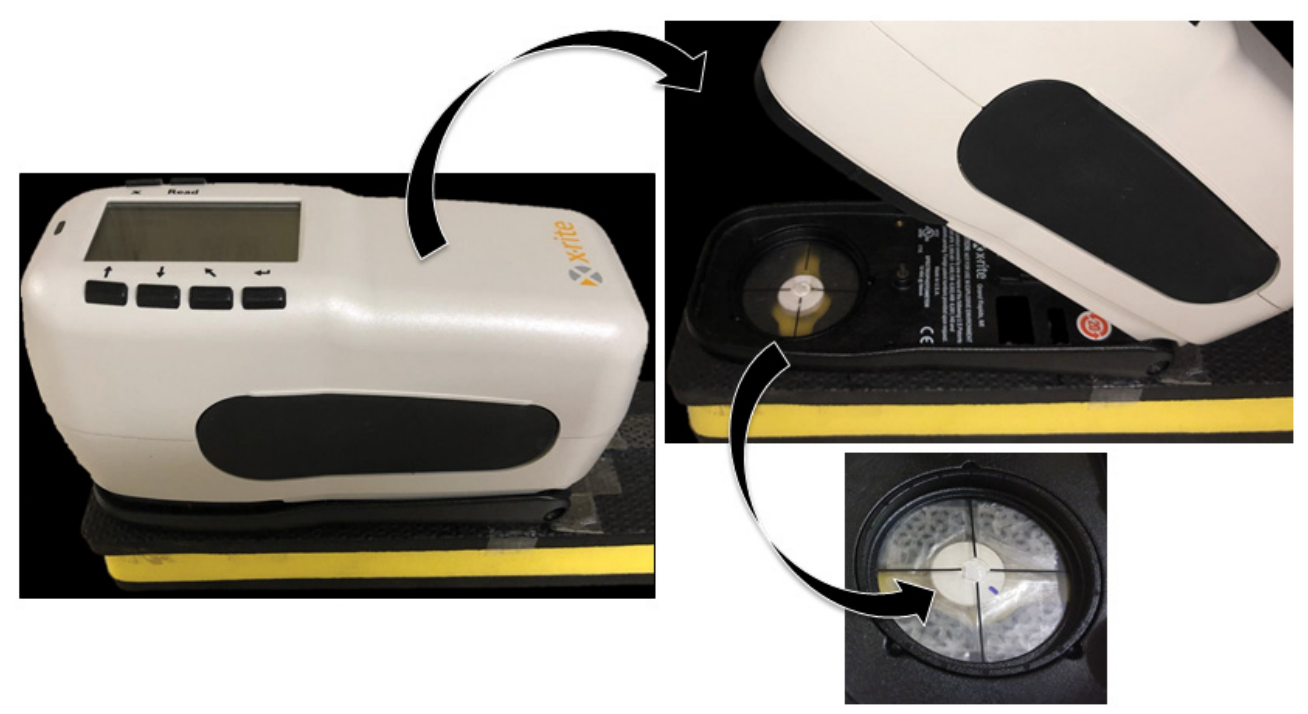

Figure 2. Color reading analysis. 


\section{STATISTICAL ANALYSIS}

The exploratory analysis showed the data do not meet the assumptions of an analysis of variance. Then, generalized linear models were adjusted considering the outline of measures repeated in time for $L^{*}, a^{*}$, and $b^{*}$ values. The variation data (deltas) were analyzed with Kruskal Wallis and Dunn's non-parametric tests. The analyses were performed in the R and SAS software (Institute Inc., Cary, NC, USA, Release 9.3, 2011) at $5 \%$ significance level.

The numerical data were analyzed with the Kruskal Wallis test at $p<0.05$. The difference of final values observed for $L^{*}, a^{*}$ and $b^{*}$ of each sample from their initial values was considered a variable. A non-parametric test was selected for the statistical analysis of the data observed in the experiment.

\section{RESULTS}

There was a statistically significant decrease in the $L^{*}$ value after the immersion in all solutions and distilled water $(p=0.0002)$. The highest $L^{*}$ value was observed in the groups with distilled water and chlorhexidine $(p<0.05)$ and the lowest values in the group with chlorhexidine associated with wine $(\mathrm{p}<0.05)$.

As for the $a^{\star}$ values, there was a significant increase after the immersion in all solutions and distilled water $(p<0.0001)$. At the final time, the highest values were observed for the groups that received wine and chlorhexidine associated with wine $(p<0.05)$, and the lowest values were observed for distilled water and chlorhexidine $(p<0.05)$.

The $b^{*}$ value also showed a significant increase at the final time relative to the initial time in all groups $(p<0.0001)$. At the final time, the highest values were observed for instant coffee and the lowest values for distilled water, chlorhexidine, and wine $(\mathrm{p}<0.05)$.

Table 1 presents the analysis of variation for $L^{*}, a^{*}$, and $b^{*}$ values $\left(\Delta L^{*}, \Delta a^{*}\right.$, and $\left.\Delta b^{*}\right)$ and total color variation $\left(\Delta \mathrm{E}^{\star}\right)$. It is observed that when immersed in chlorhexidine associated with wine, the brackets presented greater variations in the $L^{*}$ value than when immersed in chlorhexidine and distilled water $(p<0.0001)$. Except for the brackets immersed in chlorhexidine, all the other groups presented greater variation of

Table 1. Median (minimum and maximum values) of the variation of $L^{\star}, a^{\star}$, and $b^{\star}$ (delta $L$, delta $a$, and delta $b$ ) and total color (delta E) values according to solution, at the final time relative to the initial time (TFinal-TO).

\begin{tabular}{lcccc}
\hline Solution & Delta $L^{*}$ & Delta $\mathbf{a}^{*}$ & Delta b* & Delta $\mathrm{E}^{*}$ \\
\hline Distilled water & $-2.51(-8.79 ;-0.99) \mathrm{a}$ & $0.18(0.13 ; 0.29) \mathrm{bc}$ & $1.28(0.03 ; 2.74) \mathrm{c}$ & $2.89(1.00 ; 8.85) \mathrm{c}$ \\
\hline $\begin{array}{l}\text { 0.12\% } \\
\text { chlorhexidine }\end{array}$ & $-3.47(-4.93 ;-2.83) \mathrm{ab}$ & $0.16(0.01 ; 0.29) \mathrm{c}$ & $1.67(1.15 ; 2.49) \mathrm{bc}$ & $3.90(3.25 ; 5.06) \mathrm{c}$ \\
\hline Instant coffee & $-6.23(-11.50 ;-2.60) \mathrm{bc}$ & $1.06(0.68 ; 2.42) \mathrm{a}$ & $7.87(4.30 ; 11.33) \mathrm{a}$ & $9.91(5.07 ; 16.32) \mathrm{b}$ \\
\hline Red wine & $-7.16(-11.76 ;-5.81) \mathrm{c}$ & $2.09(0.77 ; 2.76) \mathrm{a}$ & $2.02(0.86 ; 5.29) \mathrm{bc}$ & $7.89(6.40 ; 13.09) \mathrm{b}$ \\
\hline $\begin{array}{l}\text { Chlorhexidine } \\
\text { and coffee }\end{array}$ & $-6.24(-9.42 ;-3.93) \mathrm{bc}$ & $1.02(0.47 ; 2.14) \mathrm{ab}$ & $6.55(3.54 ; 8.97) \mathrm{a}$ & $9.31(5.31 ; 12.95) \mathrm{b}$ \\
\hline $\begin{array}{l}\text { Chlorhexidine } \\
\text { and wine }\end{array}$ & $-14.32(-19.86 ;-4.12) \mathrm{c}$ & $2.13(0.83 ; 3.06) \mathrm{a}$ & $6.00(0.81 ; 7.58) \mathrm{ab}$ & $15.68(4.28 ; 21.32) \mathrm{a}$ \\
\hline
\end{tabular}

Medians followed by different letters vertically differed from each other $(\leq 0.05)$. 
the $a^{*}$ value when compared with the control group, which received distilled water $(p<0.05)$, according to Table 1 . The groups that received instant coffee, chlorhexidine associated with coffee, and chlorhexidine associated with wine presented greater variation in the $b^{*}$ value than the control group ( $\left.p=0.0007\right)$. Table 1 also shows that $\Delta E^{*}$ (color variation) was greater in the group that received chlorhexidine associated with wine and lower in the groups that received distilled water and chlorhexidine $(p<0.05)$.

\section{DISCUSSION}

In this study, the null hypothesis was rejected because that chlorhexidine potentiates staining when associated with dye substances, and chlorhexidine associated with red wine promotes greater color change than the other substances and associations tested.

In dentistry, the antibacterial agents can perform a bactericidal action that is rather important for controlling bacterial colonies, thus safely promoting anticariogenic benefits. In this context, the current gold standard of antibacterial agent is chlorhexidine, because it is a cationic detergent from the class of bisbiguanides and available in the forms of acetate, hydrochloride, and gluconate. The latter is the salt most commonly used in formulas and products ${ }^{20,21}$. However, chlorhexidine has some undesirable effects such as the appearance of extrinsic coloration in tooth enamel.

In the present study, the polycrystalline ceramic brackets were subjected to treatments with chlorhexidine associated or not with dye substances, which were previously used in studies on staining 9 .10.

The spectrophotometer measures a wavelength by time from the reflective property or object transfer ${ }^{22}$. This study assessed the samples immersed in staining solution for three minutes under agitation, once a day for five days of the week, during 28 days, according to the protocol used by Godoi et al. ${ }^{16}$ (2011). These 28 days represent clinically 4 indications of use of this mouthwash for 5 days. It is noteworthy that these 4 indications are present within the average clinical time of orthodontic treatment which is approximately 1.5 years.

The $L^{*}, a^{*}$, and $b^{*}$ values were used for each bracket, in which $L^{*}$ corresponds to luminosity and $a^{\star}$ and $b^{*}$ correspond to chromaticity. If $L^{*}$ is positive, the color will change toward white and if negative, the color will change toward black. Positive $a^{\star}$ changes toward red and negative $a^{\star}$ toward green, while positive $b^{\star}$ changes toward yellow and negative $b^{*}$ toward blue. Color change is obtained with the following calculus: $\Delta E^{\star}=\left[\left(\Delta L^{*}\right)^{2}+\left(\Delta a^{\star}\right)^{2}+\left(\Delta b^{\star}\right)^{2}\right]^{1 / 22}$. The $\Delta E^{\star}$ value considers the total color change relative to the $L^{\star}, a^{\star}$, and $b^{*}$ axes. According to a previous study, if $\Delta E^{\star}<1.0$, color change is nor visibly perceptible; if $1<\Delta E^{*}<3.3$, color change is considered clinically acceptable; and if $\Delta E^{*}>3.3$, color change has clinical significance ${ }^{23}$.

Several hypotheses were investigated for the mechanisms associated with staining. This finding may be explained by the fact that the cationic molecule of chlorhexidine is rapidly attracted by the negative load of the cell membrane and it is adsorbed to the cell membrane by electrostatic interactions, potentially by hydrophobic ligation or hydrogen bridges. Such adsorption is concentration-dependent. Thus, at high doses, it causes the precipitation and coagulation of cytoplasmic proteins and bacterial death. At low doses, the integrity of the cell membrane changes, resulting in an over- 
flow of the bacterial components of low molecular weight ${ }^{24}$. This study showed that among the $\Delta \mathrm{E}^{\star}$ (color variation) results, the group of chlorhexidine associated with wine presented statistical difference when compared with other experimental groups. The explanation of the potentiation of chlorhexidine staining when associated with chromogenic foods may lie in the fact that cationic mouthwashes such as chlorhexidine may precipitate or ligate to anionic dyes contained in foods and beverages ${ }^{25}$.

When considering the $\Delta L^{*}$, the groups of distilled water and chlorhexidine did not show statistically significant difference $(p<0.05)$. Despite the little influence on color change, water seems to change the brightness of brackets, even though such change is not significant, because the color change is considered clinically acceptable ${ }^{12}$.

The groups of chlorhexidine and chlorhexidine associated with coffee did not present statistical difference in value when compared with the control group that received distilled water $(p<0.05)$. Groups of instant coffee, red wine, and chlorhexidine associated with red wine were likely to increase the red color with greater variation of the $a^{\star}$ value, which may occur due to the excessive consumption of red wine, causing extrinsic pigmentation due to the high content of tannin compounds that are highly denaturing ${ }^{26}$. Similarly, a previous study showed higher staining potential in red wine, because its composition contains a high concentration of pigments and it presents low $\mathrm{pH}$ and a reasonable amount of alcohol ${ }^{27}$.

The groups that received instant coffee, chlorhexidine associated with coffee, and chlorhexidine associated with wine presented greater variation for the $b^{\star}$ value than the control group $(p<0.05)$ and they were likely to increase the yellow color, which agrees with a previous study that observed that the yellow pigment in coffee is less polar and therefore less hydrophilic. In studies with resin composites, the discoloration by coffee is processed by absorption and adsorption of polar dyes, potentially due to the compatibility of the organic phase of the polymer with this specific dye ${ }^{28}$, and the staining increased when the surface of the specimen tested was subjected to the use of chlorhexidine.

The group chlorhexidine associated with red wine has potentiated the effect of the staining in relation to the other substances, because the red wine has in its composition a tannin, a polyphenol, with high molecular weight, generally between 500 and 3000 daltons, soluble in water and in addition, may still be favored by the use of cationic antiseptics such as chlorhexidine ${ }^{29}$, precipitating or bind to anionic coloring agents contained in foods and beverages ${ }^{30}$.

In studies with ceramic brackets, in which the major component is inorganic, the literature has not yet explained the interaction among dyes from solutions and the components of such materials. Hence, the development of further studies in this field would be important. Whitin this, it is concluded that chlorhexidine potentiates the staining caused by red wine in polycrystalline ceramic brackets.

\section{REFERENCES}

1. Lee $Y-K$, Bin $Y$. Translucency and color match with a shade guide of esthetic brackets with the aid of a spectroradiometer. Dent Press J Orthod. 2016 Mar-Apr;21(2):81-7. doi: 10.1590/2177-6709.21.2.081-087.oar. 
2. dos Santos PR, Meneghim MC, Ambrosano GM, Vedovello Filho M, Vedovello SA Influence of quality of life, self-perception, and self-esteem on orthodontic treatment need. Am J Orthod Dentofacial Orthop. 2017 Jan;151(1):143-7. doi: 10.1016/j.ajodo.2016.06.028.

3. Gkantidis N, Zinelis S, Karamolegkou M, Eliades T, Topouzelis N. Comparative assessment of clinical performance of esthetic bracket materials. Angle Orthod. 2012 Jul;82(4):691-7. doi: 10.2319/092511-605.

4. Kukiattrakoon B, Samruajbenjakul B. Shear bond strength of ceramic brackets with various base designs bonded to aluminous and fluorapatite ceramics. Eur J Orthod. 2010 Feb;32(1):87-93.

5. Alexopoulou E, Polychronis G, Konstantonis D, Sifakakis I, Zinelis S, Eliades T.

A study of the mechanical properties of as-received and intraorally exposed single-crystal and polycrystalline orthodontic ceramic brackets. Eur J Orthod. 2019 Apr 23. pii: cjz024. doi: 10.1093/ejo/cjz024.

6. Maltagliati LA, Feres R, Figueiredo MA, Siqueira DF. [Aesthetic brackets: clinical considerations] Rev Clín Ortod Dental Press. 2006 Jun-Jul;5(3):89-95. Portuguese.

7. Karamouzos A, Athanasiou AE, Papadopoulos MA. Clinical characteristics and properties of ceramic brackets: a comprehensive review. Am J Orthod Dentofacial Orthop. 1997 Jul;112(1):34-40.

8. Oliveira CB, Maia LG, Santos-Pinto A, Gandini Júnior LG. In vitro study of color stability of polycrystalline and monocrystalline ceramic brackets. Dental Press J Orthod. 2014 Jul-Aug;19(4):114-21.

9. Guignone BC, Silva LK, Soares RV, Akaki E, Goiato MC, Pithon MM, et al. Color stability of ceramic brackets immersed in potentially staining solutions. Dental Press J Orthod. 2015 Jul-Aug;20(4):32-8. doi: 10.1590/2176-9451.20.4.032-038.oar.

10. Lima Filho HL, Maia LH, Araújo MV, Eliast CN, Ruellas AC. Colour stability of aesthetic brackets: ceramic and plastic. Aust Orthod J. 2013 May;29(1):13-20.

11. Vivek Aithal PR, Akshai Shetty KR, Dinesh MR, Amarnath BC, Prashanth CS, Roopak MD. In vitro evaluation of microbial contamination and the disinfecting efficacy of chlorhexidine on orthodontic brackets. Prog Orthod. 2019 May 1;20(1):17. doi: 10.1186/s40510-019-0270-4.

12. Mendonça MR, Fabre AF, Goiatto MC, Cuoghi OA, Martins LP, Verri ACG. Spectrophotometric evaluation of color changes of esthetic brackets stored in potentially staining solutions. RPG Rev Pos Grad. 2011;18(1):20-7.

13. Gürdal P, Akdeniz BG, Hakan Sen B. The effects of mouthrinses on microhardness and colour stability of aesthetic restorative materials. J Oral Rehabil 2002 Sep;29(9):895-901.

14. Mathur S, Tanu M, Rahul S. Chlorhexidine: The gold standard in chemical plaque control. Natl J Physiol Pharm Pharmacol. 2011;1(2):45-50.

15. Colucci V, Dos Santos CD, Amaral FL, Corona SA, Catirse AB. Influence of $\mathrm{NaHCO}$ powder on translucency of microfilled composite resin immersed in different mouthrinses. J Esthet Restor Dent. 2009;21(4):242-8. doi: 10.1111/j.1708-8240.2009.00269.x.

16. Godoi APT, Freitas DB, Trauth KGS, Colucci V, Catirse AB. Combined effect of the association between chlorhexidine and a diet protein on color stability of resin composites. Int J Clin Dent. 2011 Jan;4(3):113-121.

17. Nagem Filho H, D'Azevedo MTFS, Nagem HD, Marsola FP. Surface roughness of composite resins after finishing and polishing. Braz Dent J 2003;14(1):37-41.

18. Brandão RB, Martin C, Catirse ABCE, De Castro e Silva M, Evison MP, Guimarães MA. Heat induced changes to dental resin composites: a reference in forensic investigations? J Forensic Sci. 2007 Jul;52(4):913-9. 
19. Cui T, Luo W, Xu L, Yang B, Zhao W, Cang H. Progress of Antimicrobial Discovery Against the Major Cariogenic Pathogen Streptococcus mutans. Curr Issues Mol Biol. 2019 Jun 5;32:601-44. doi: $10.21775 /$ cimb.032.601.

20. Zanatta FB, Rosing CK. Chlorhexidine: action's mechanisms and recent evidences of its efficacy over supragingival biofilm context. Scientific-A 2007;1(2):35-43.

21. Joiner A. Tooth colour: a review of the literature. J Dent. 2004;32(Suppl 1):3-12.

22. Guignone BC, Silva LK, Soares RV, Akaki E, Goiato MC, Pithon MM, et al. Color stability of ceramic brackets immersed in potentially staining solutions. Dental Press J Orthod. 2015 Jul-Aug;20(4):32-8. doi: 10.1590/2176-9451.20.4.032-038.oar.

23. Hjeljord LG, Rolla G, Bonesvoll P. Chlorhexidine-protein interactions. J Periodontal Res Suppl. 1973;12:11-6.

24. Leard A, Addy M. The propensity of different brands of tea and coffee to cause staining associated with chlorhexidine. J Clin Periodontol. 1997 Feb;24(2):115-8.

25. Norbdo H. Discoloration of dental pellicle by tannic acid. Acta Odontol Scand. 1977;35(6):305-10.

26. Bagheri R, Burrow MF, Tyas M. Influence of food-simulating solutions and surface finish on susceptibility to staining of aesthetic restorative materials. J Dent. 2005 May;33(5):389-98.

27. Um CM, Ruyter IE. Staining of resin-based veneering materials with coffee and tea. Quintessence Int. 1991 May;22(5):377-86.

28. Pontefract H, Courtney M, Newcombe RG, Addy M. Development of methods to enhace extrinsic tooth discoloration for comparison of toothpaste. 1. Studies in vitro. J Clin Periodontol. 2004 Jan;31(1):1-6.

29. Lee YK, Powers JM. Combined effect of staining substances on the discoloration of esthetic Class $V$ dental restorative materials. J Mater Sci Mater Med. 2007 Jan;18(1):165-70.

30. Shree Roja RJ, Sriman N, Prabhakar V, Minu K, Subha A, Ambalavanan P. Comparative evaluation of color stability of three composite resins in mouthrinse: an in vitro study. J Conserv Dent. 2019 Mar-Apr;22(2):175-80. doi: 10.4103/JCD.JCD_241_18. 